\title{
Erfahrungen mit dem Technicon SMA 12-60 Analysengerät, on-line an einen Prozeßrechner IBM 1800 angeschlossen, im Vergleich zum Technicon SMA 12-30
}

\author{
Von R. M. Schmüling, H. Liebich, M. Locher, I. Mildner und M. Eggstein \\ Aus der Mediziniscben Universitätsklinik Tübingen Abteilung IV \\ (Vorstand: Prof. Dr. M. Eggstcin)
}

(Eingegangen am 4. April/23. Juli 1973)

\author{
Herrn Professor Dr. med. Dr. med. b. c. Hans Erbard Bock zum 70. Geburtstag genidmet
}

\begin{abstract}
Aus mehrjähriger Erfahrung wurde für die beiden Technicongeräte SMA 12-30 und SMA $12-60$ ein Analysenspektrum zusammengestellt und ein Beschickungsschema entwickelt. Die tatsächlich erreichte Analysenfrequenz wird als Effektivität der Probenahmefrequenz gegenübergestellt. Die Präzision der Methoden in der unterbrochenen Serie, die durch Definition von der Präzision von Tag zu Tag abgegrenzt wird, zeigt die Fortentwicklung des SMA 12-60 gegenüber dem Vorgängertyp, aber auch die Nachteile. Die Anfälligkeit der Methoden wird definiert und quantifiziert den Fehlermöglichkeiten durch den on-line Anschluß an einen Prozeßrechner gegenübergestellt. Die Ursachen der methodischen Fehler werden zahlenmäßig aufgeschlüsselt.
\end{abstract}

\section{Experiences with the Tecbnicon SMA 12-60 on-line connected to an IBM 1800 process computer in comparison to SMA 12-30}

On the basis of several years of experience, a spectrum of analyses and a sample feeding scheme were developed for both the technicon SMA (Sequential Multiple Autoanalyzer) $12-30$ and the SMA 12-60. The actual analysis rate defined as effectivity is compared to the sampling rate. The precision of the methods in interrupted series differentiated from the precision from day to day demonstrates the development and advantages and the disadvantages of the SMA 12-60 compared to the older instrument. The liability of the methods is defined, quantified and compared to failures due to the on-line connection to the process computer. The causes for methodical failures are classified.

Drei Jahre war im klinisch-chemischen Laboratorium der Medizinischen Universitätsklinik Tübingen ein Analysengerät Technicon SMA 12-30 eingesetzt. Es wurde im Januar 1971 durch den Typ SMA 12-60 ersetzt. Die Geräte sind mit einem Turntable IBM 1894 zur permanent wirksamen Probenidentifizierung ausgerüstet.

Beim Austausch lag ein Vergleich der Bedienungs- und chemisch-technischen Eigenschaften der beiden Geräte untereinander nahe. Dabei waren die gerätebedingten Fehler in Analysen- und Identifizierungsfehler zu unterscheiden. Durch den on-line-Anschluß ist eine direkte Übernahme der Analogwerte vom Schreiber des Gerätes und eine Digitalwertübernahme aus dem Lesegerät für Kurzlochkarten durch den Prozeßrechner gewährleistet. Die lückenlose, von konventionellen Datenträgern unabhängige Übertragung und Zuordnung von Analog- und Identifizierungssignalen aus vollmechanisierten Analysengeräten in einen Rechner stellt zur Zeit die beste Lösung dieser Informationsübermittlung dar.

SMA $12-30$ wie $12-60$ sind in unserem Laboratorium auf das gleiche Untersuchungsspektrum festgelegt, welches ein Kompromiß zwischen großem Bedarf bestimmter Analysen, präventivmedizinischen Aspekten und methodischen Möglichkeiten bildet. Bestimmt werden Kreatinin, Harnstoff, Harnsäure, Phosphor, Calcium, Chlorid, Gesamteiweiß, Albumin, Choleste- rin, Bilirubin und Serumzucker, Methoden, die vom Gerätehersteller bis auf geringfügige Änderungen übernommen wurden. Beim SMA 12-30 wurde das Flußschema für Bilirubin und Kreatinin gegenüber dem Originalschema variiert, beim SMA 12-60 wurden keine methodischen Veränderungen gegenüber den Empfehlungen des Herstellers vorgenommen.

\section{Beschickungsschema des SMA 12}

Die Justierung und Kontrolle der Geräte folgt einem festgelegten Beschickungsschema, das auf den Erfahrungen mit dem SMA $12-30$ beruht $(1,2)$. Es berücksichtigt die Probenidentifizierung, welche durch den Proben anhängende Kurzlochkarten gewährleistet wird. (Tab. 1)

Die Gerätejustierung vor der täglichen Inbetriebnahme umfaßt die Einstellung der Basis, die Phasing-Kontrolle und die Kalibrierung anhand von 15 Positionen (I. II. III. in Tab. 1), bevor die erste Analyse eines Patientenserums anfällt. Auf die Justierung folgen die angeforderten Proben, die in Blöcken zu 20 Patientenseren zusammengefaßt sind. Daran schließen sich jeweils 5 Positionen an, die der Kontrolle und Nachregulierung des Gerätes dienen (IV. in Tab. 1). Erst dieses Schema gewährleistet eine ausreichende Úberwachung des Gerätes und garantiert die von uns erreichte Präzision bei noch vertretbarer Einschränkung der realen Analysenfrequenz. 
Tab. 1

Beschickungsschema des Technicon SMA 12

SMA $12-60$

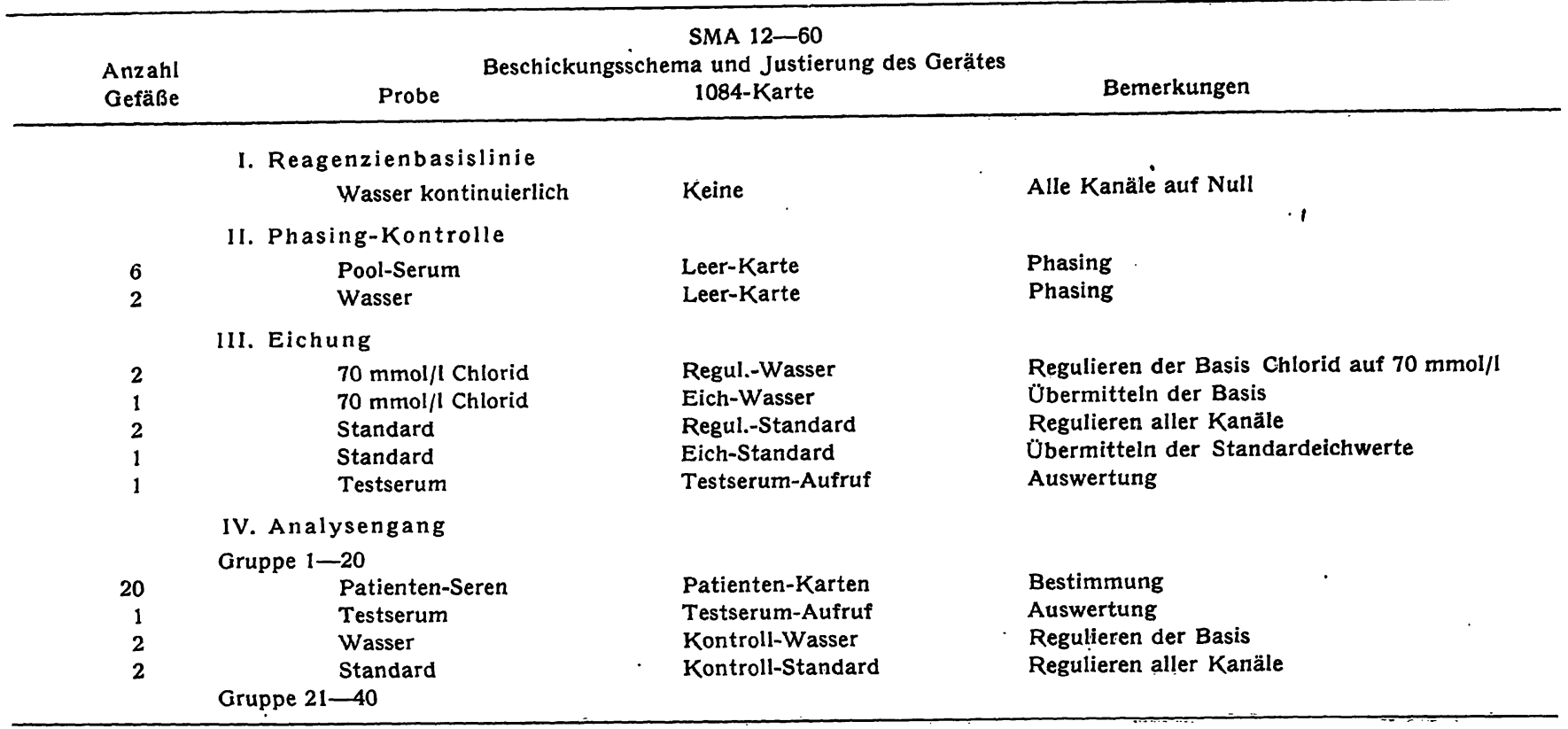

usw.

Analysenfrequènz im SMA 12-30 und 12-60

Die von uns erreichten Untersuchungsproben pro $h$ decken sich nicht mit der vom Hersteller angegebenen Probenfrequenz von 30 bzw. 60 pro $h$. Die Effektivität der Geräte - wir verstehen damit den Prozentsatz von gewünschten Bestimmungen in $\mathrm{Pa}$ tientenseren an der Gesamtheit der dafür notwendigen Bestimmungen einschließlich der Kontrollpositionen geht aus Tabelle 2 hervor. Etwa $30 \%$ bzw. $25 \%$ von 100 bzw. 200 Bestimmungen dienen der Kontrolle und Justierung der Geräte.

Bevor der SMA 12-30 oder 12-60 die ersten Analysenergebnisse von Proben liefert, vergeht eine Vorbereitungszeit von im Durchschnitt $2 \mathrm{~h}$. Dann folgt die reine Durchlaufzeit von 140 bzw. 265 Positionen, entsprechend 100 bzw. 200 vorgelegten Patientenproben, unter der Voraussetzung, daß kein technisches Versagen zu einem Unterbrechen des Arbeitsflusses. zwingt.

Effektiv schafft ein SMA $12-30$ also zwischen 15 und 19, ein SMA 12-60 zwischen 23 und 31 Proben bzw. Patientenseren pro Arbeitsstunde (Tab. 2), wobei pro Probe (Serum) 11 bzw. 12 Einzelwerte anfallen.

Tab. 2

Effektive Leistung von SMA 12-30 und 12-60

\begin{tabular}{lcccc}
\hline & \multicolumn{2}{c}{ SMA } & $12-30$ & \multicolumn{2}{c}{ SMA $12-60$} \\
\hline Patientenseren & 100 & 200 & 100 & 200 \\
Kontrollpositionen & 40 & 65 & 40 & 65 \\
Gesamtpositionen & 140 & 265 & 140 & 265 \\
$\begin{array}{l}\text { Effektivität } \\
\text { Vorbereitungszeit }\end{array}$ & $71,4 \%$ & $75,5 \%$ & $71,4 \%$ & $75,5 \%$ \\
$\quad \begin{array}{l}\text { (h) } \\
\text { Laufzeit dafür in } \\
\quad \text { h }\end{array}$ & 2 & 2 & 2 & 2 \\
$\begin{array}{l}\text { Patientenseren effektiv } \\
\text { pro Arbeitsstunde }\end{array}$ & 15,0 & 18,5 & 23,0 & 31,2 \\
\hline
\end{tabular}

Diese Berechnung berücksichtigt keine Auswert- und Registrierarbeit. Sie wird beim on-line-Anschluß an einen Rechner in dem von uns gewählten Organisationssystem tatsächlich auch überflüssig.

Der Personalbedarf beträgt 1,5 med. techn. Assistenzkräfte. Eine Kraft ist mit der Gerätebedienung, mit dem Ansetzen von Lösungen und der Kontrolle der Resultate anhand von Vorwerten (Arbeitsliste vgl. 3) beschäftigt. Eine halbe Kraft zentrifugiert die eingehenden Seren und beschickt den Turntable mit den Proben und anhängenden Kurzlochkarten.

Die folgende Tabelle 3 belegt diese Kalkulationen anhand einer $34 \mathrm{bzw}$. 45 Tage umfassenden Bilanz. (Die Zahlen entstammen zum Teil einem von Frl. Dipl. Phys. I. Mieth erstellten Programm zur wöchentlichen Statistik der Arbeitsbelastung.) Im angegebenen Zeitraum wurden mit dem SMA 12-30 durchschnittlich pro Tag 117 und mit dem SMA 12-60 1'32 Patientenseren untersucht. Bei $126 \mathrm{bzw} .210$ theoretisch möglichen Proben laut den Berechnungen der Tabelle 2 bedeutet das eine Auslastung der Kapazität des SMA

Tab. 3

Durchschnittliche Arbeitsleistung pro Tag

\begin{tabular}{lcc}
\hline & SMA 12-30 & SMA 1.2-60 \\
\hline Zeitraum & XI.-XII. 1970 & IX.-XI. 1971 \\
Arbeitstage & 34 & 45 \\
Patientenseren & 117 & 132 \\
$\begin{array}{l}\text { Bestimmungen } \\
\text { Bestimmungen pro } \\
\quad \text { Patientenserum }\end{array}$ & 1251 & 1436 \\
$\begin{array}{l}\text { Positionen einschließlich } \\
\quad \text { Kontrollen und Testseren }\end{array}$ & 10,6 & 10,9 \\
$\begin{array}{l}\text { Arbeitszeit dafür (h) } \\
\text { Bei 8 h mögliche } \\
\text { Patientenserumbe- }\end{array}$ & 7,40 & 182 \\
stimmungen & 126 & 5,04 \\
\hline
\end{tabular}


12 -30 von etwa $93 \%$ und des SMA $12-60$ von $63 \%$. Diese Belastung des SMA 12-30, die bei der geringsten Störung ein Überziehen der normalen Arbeitszeit notwendig machte - im Diagnostik-InformationsSystem Tübingen wegen des ausgeklügelten Zeitplanes besonders folgenschwer - rechtfertigte die Anschaffung des schnelleren SMA 12-60. Dessen Kapazität ist nun zum Zeitpunkt der Einreichung dieser Arbeit bis zu 70\% ausgeschöpft.

Bei beiden Geräten liegt die durchschnittliche Zahl der in jeder Probe ermittelten Meßpunkte unter den 11 theoretisch möglichen. Dafür verantwortlich sind Fehlbestimmungen in einzelnen Kanälen und Bestimmungen in verdünnten Seren zur Ermittlung stark erhöhter Werte, die bei der von uns gewählten Geräteeinstellung keine relevanten Chloridwerte liefern.

Der SMA $12-60$ braucht $1,8 \mathrm{ml}$ Serum pro Bestimmung, der Vorgänger $2,8 \mathrm{ml}$, weshalb mit dem neuen Gerät häufiger Kontrollbestimmungen vorgenommen werden können, so daß bei 10 Untersuchungsspektren im Durchschnitt 1 Ergebnis fehlt gegenüber 4 Ausfällen beim SMA 12-30.

Präzision der Analysen im SMA 12-60 und 12-30

Richtigkeit und Präzision der Bestimmungsmethoden wird auf beiden Geräten durch das regelmäßige Einfügen eines Testserums, wie aus dem Beschickungsschema ersichtlich, überprüft.

Als Testserum wird eine Serumzubereitung (Lyophilisat oder stabilisierte Lösung) bezeichnet, deren Konstituenten in ihrer Konzentration bekannt sind und zwar durch

1. Einwaage,

2. Konzentrationsermittlung mit voneinander unabhängigen Methoden,

3. Vergleiche mit primären Standards im Sinne von RADIN (4),

4. Angaben der Hersteller.

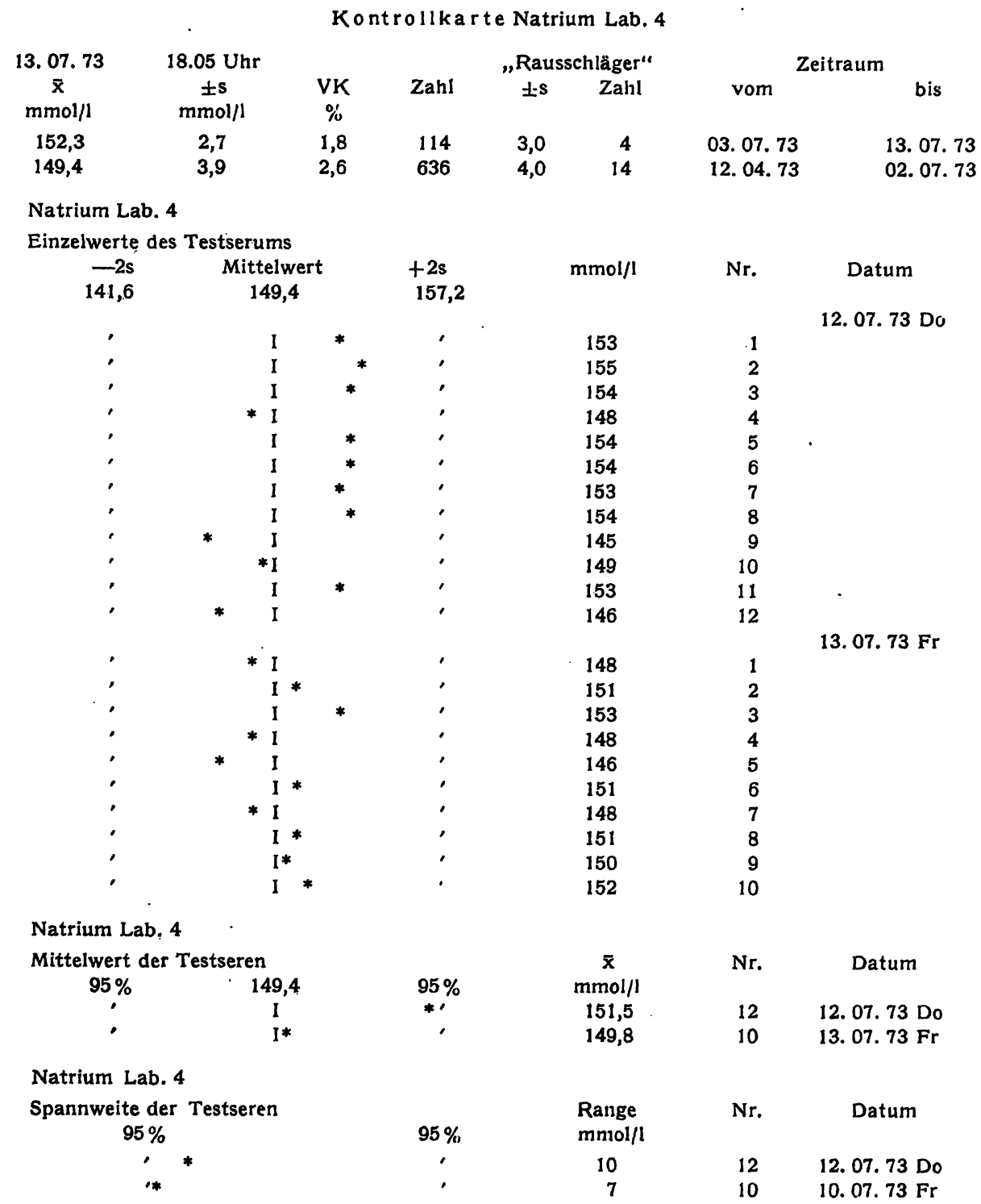

Abb. 1 
(Die folgenden Angaben zur Präzision basieren auf der Benutzung von Hyland Controlserum, Dried, als Testserum. Die Richtigkeit der Methoden wird zum weiteren durch Lösungen mit bekannter Konzentration überprüft, die den mit dem Leben zu vereinbarenden Konzentrationsbereich absichern.)

\section{Kontrollkarten}

Im Diagnostik-Informations-System(D-I-S) war der SMA 12-30 und ist der SMA $12-60$ on-line an einen Prozeßrechner IBM 1800 angeschlossen (3). Dieser benutzt die Analogsignale zur Umrechnung in Konzentrationen und die Digitalsignale (Patienten-Nummer) zur Identifizierung der Bestimmung. Berechnung und Identifizierung werden von einem sogenannten Auswertprogramm vorgenommen. In die Auswertprogramme für die verschiedenen Laborgeräte ist jeweils ein Unterprogramm für die Erkennung und separate Speicherung der Testserumergebnisse eingebaut. Auf einem speziellen Datenbereich gespeichert, stehen die Testserumergebnisse zur sofortigen statistischen Auswertung bereit, ihre Integrität ist gesichert.

Ihre Dokumentation erfolgt gemäß Abbildung 1. Sie läßt 3 Abschnitte erkennen

1. Grafische Auswertung aller Testserumwerte,

2. Tagesmittelwerte und

3. Spannweiten der Testseren pro Tag.

Nach diesem Prinzip erfolgt die Überwachung der Präzision aller im Laboratorium on-line angeschlossenen Geräte. Im wesentlichen folgen wir bei der Beurteilung den Vorschlägen von HaLd (5) und BenNETT-FrankLIN (6).

Präzision in der Serie für Messungen mit dem SMA $12-60$ und $12-30$

Als „Präzision in der Serie“ wird der Variationskoeffizient für unmittelbar aufeinander folgende Bestimmungen der selben Substanz, in diesem Fall von Serum, verstanden.

Die im SMA 12 unter diesen Bedingungen errechneten Parameter sind in Tabelle 4 zusammengestellt. Die Meßverfahren für Kreatinin, Harnsäure, Phosphor, Gesamteiweiß und Serumzucker des SMA 12-60 liefern eine (im F-Test ermittelte) signifikant bessere Präzision in der Serie, das heißt einen kleineren VK als die des SMA 12-30. Relevant ist die Verbesserung nur bei den Bestimmungsmethoden von Kreatinin, Phosphor und Harnsäure. Die Bestimmung des Bilirubin läßt im vorliegenden Stichprobenumfang keine Beurteilung $\mathrm{zu}$, da die zu. erwartenden Schwankungen unter der Auflösungsgrenze liegen., ,

Die Präzision in der Serie der übrigen Methoden zeigen keinen signifikanten Unterschied zwischen den beiden Geräten bei diesem Stichprobenumfang.

Die Präzision von Tag zu Tag bzw. die Präzision in der unterbrochenen Serie

Die „Präzision von Tag zu Tag“ errechnet sich aus Testserumwerten, die an verschiedenen Tagen anfallen.

Seit der Einführung vollmechanisierter Analysengeräte mit entsprechend großer täglicher Analysenfrequenz werden pro Arbeitstag mehrere, meist in periodischen Abständen eingeschobene Testseren analysiert. Bei der Ermittlung der Präzision von Tag zu Tag bieten sich bei diesem Arbeitsschema verschiedene Zahlenkollektive für die Berechnung an.

Die Präzision von Tag zu Tag kann errechnet werden aus

1. Jeweils einem Testwert mit definierter Position aus dem Tageskollektiv,

2. Aus einem Wert mit zufälliger Position im Tageskollektiv,

3. Durch Berücksichtigung mehrerer, aber in der Zahl konstant festgelegter Testseren im Tageskollektiv,

4. Durch Berücksichtigung aller im Verlauf eines jeden Arbeitstages erhobenen Testserumwerte.

Wir gehen nach Punkt 4 vor, nachdem, wie Tabelle 5 zeigt, der nach diesem Schema errechnete VK sich nicht signifikant von nach Schema 1 ausgewählten Kenngrößen zur Ermittlung der Präzision unterscheidet. Wir bezeichnen die so gewonnenen Parameter als Präzision in der unterbrochenen Serie, um deutlich zu machen, daß ein formaler Unterschied zur Präzision von Tag zu Tag besteht.

Tab. 4

Gegenüberstellung der Präzision in der Serie

\begin{tabular}{|c|c|c|c|c|c|c|c|c|}
\hline & \multicolumn{4}{|c|}{$\begin{array}{c}\text { SMA } 12-60 \\
n=15\end{array}$} & & \multicolumn{3}{|c|}{$\underset{n=21}{S M A} 12-60$} \\
\hline & & $\overline{\mathbf{x}}$ & $\mathbf{s}$ & VK \% & & VK \% & $\mathbf{s}$ & $\ldots \overline{\mathbf{x}}$ \\
\hline 2. Harnstoff & {$[\mathrm{mmol} / \mathrm{l}]$} & 12,1 & 0,1 & 0,8 & & 0,4 & 0,1 & 24,5 \\
\hline 3. Harnsäure & {$[\mathrm{mmol} / \mathrm{l}]$} & 0,43 & 0,02 & 3,9 & $>$ & 0,7 & 0,002 & 0,38 \\
\hline 4. Phosphor & {$[\mathrm{mmol} / \mathrm{l}]$} & 1,43 & 0,02 & 1,1 & $>$ & 0 & 0 & 1,94 \\
\hline 5. Calcium & {$[\mathrm{mmol} / \mathrm{l}]$} & 3,27 & 0,03 & 0,8 & & 1,0 & 0,03 & 2,49 \\
\hline 7. Gesamteiweiß & {$[\mathrm{g} / 1]$} & 75 & 1,1 & 1,5 & $>$ & 0,8 & 0,6 & 68,3 \\
\hline 8. Albumin & {$[\mathrm{g} / \mathrm{l}]$} & 40,1 & 0,6 & 1,5 & & 1,2 & 0,5 & 38,3 \\
\hline 9. Cholesterin & {$[\mathrm{mmol} / \mathrm{l}]$} & 5,85 & 0,10 & 1,7 & & 1,6 & 0,09 & 5,28 \\
\hline 10. Bilirubin & {$[\mathrm{mmol} / 1]$} & 0,012 & $=0$ & 0 & $<$ & 3,2 & 0,001 & 0,031 \\
\hline 11. Glucose im Serum & {$[\mathrm{mmol} / \mathrm{l}]$} & 15,4 & 0,1 & 0,9 & $\stackrel{p}{>0,05}$ & 0,5 & 0,07 & 14,4 \\
\hline
\end{tabular}


Tab. 5

Gegenüberstellung Präzision von Tag zu Tag und Präzision in der unterbrochenen Serie

\begin{tabular}{ccccc}
\hline $\begin{array}{c}\text { Kalium } \\
\text { Stelle }\end{array}$ & $\overline{\mathrm{x}}$ mmol/1 & s mmol/1 & VK\% & $\mathrm{n}$ \\
\hline 1. & 4,73 & 0,20 & 4,3 & 26 \\
2. & 4,73 & 0,16 & 3,4 & 26 \\
3. & 4,70 & 0,18 & 3,8 & 26 \\
4. & 4,76 & 0,19 & 4,1 & 26 \\
5. & 4,75 & 0,14 & 3,1 & 26 \\
6. & 4,72 & 0,15 & 3,1 & 26
\end{tabular}

Aus allen Werten vom 31. 10. 1969 bis 8. 12. 1969

$$
4,73 \quad 0,18 \quad 3,8
$$

287

Betrachtet wird in Tabelle 5 ein Zeitraum von 26 Arbeitstagen ( $\mathrm{n}$ ). In Zeile 1 stehen die Parameter der Testseren, die jeweils als erste pro Tag bestimmt wurden, in Zeile 2 der Testseren, die als zweites Testserum pro Tag bestimmt wurden usw. In der letzten Zeile sind dann alle Werte zu einem Kollektiv vereinigt und die Parameter gemäß der unter 4 erfolgten Definition berechnet worden.

Aus der Tabelle geht beim Vergleich der VK im FTest hervor, daß für unsere Belange und für die geprüfte Methode die Präzision von Tag zu Tag und die Präzision in der unterbrochenen Serie numerisch gleich groß sind.

Eine Gegenüberstellung der Präzision in der unterbrochenen Serie von SMA 12-30 und SMA 12-60 mit den zugehörigen Parametern zeigt die Tabelle 6.

Auch bei diesem großen Stichprobenumfang von n $>400$ zeigen 4 Methoden keinen signifikanten Unterschied an beiden Geräten und zwar Harnstoff (Tab. 6,2), Calcium (Tab. 6,5), Albumin (Tab. 6,8) und Bilirubin (Tab. 6,10).

Sechs Methoden wurden am SMA 12-60 gegenüber dem SMA 12-30 in ihrer Präzision verbessert. Es handelt sich um Kreatinin (Tab. 6,1), Harnsäure (Tab. 6,3), Phosphor (Tab. 6,4), Chlorid (Tab. 6,6), Gesamteiweiß (Tab. 6,7) und Serumzucker (Tab. 6,11). Die Signifikanz der Uńterschiede der Standardabweichungen wurde im $\mathrm{F}$-Test geprüft.

Eine wesentliche Verbesserung betrifft die Kreatininmethode mit einer Abnahme des Variationskoeffizienten von 4,4 auf $2,7 \%$. Damit ist diese Methode in bezug auf die Präzision in einen annehmbaren Bereich gelangt. In der gleichen Größenordnung liegt die Verbesserung der Serumzucker- und Phosphorbestimmung.

Eine wesentliche Verschlechterung ist bei der Cholesterinmethode des SMA 12-60 zu verzeichnen, deren Variationskoeffizient bei der Präzision in der unterbrochenen Serie von 2,9\% beim SMA 12-30 auf 4,8\% beim SMA 12-60 angestiegen ist.

\section{Anfälligkeit der Bestimmungen mit dem SMA} $12-60$

Die Präzision in der unterbrochenen Serie beziehungsweise von Tag zu Tag charakterisiert den, Analysenausschuß einer Methode bei großer täglicher Probenfrequenz nicht ausreichend. Wir empfehlen zur $\mathrm{Ab}-$ schätzung dieser methodenspezifischen Eigenschaft die Angabe der ,Anfälligkeit' einer Methode.

Als Anfälligkeit definieren wir das Verhältnis von Bestimmungen ohne verwertbares Ergebnis zur Anzahl der insgesamt durchgeführten Bestimmungen, also den Prozentsatz vergebens durchgeführter Bestimmungen, wie in Tabelle 7 zusammengestellt. Sie umfaßt 9660 Serumbestimmungen von Patienten, entsprechend 104860 Meßergebnissen, die in 3 Monaten, das sind 70 Arbeitstage, erstellt wurden.

Tab. 7

Anfälligkeit der Methoden: methodisch-gerätebedingte und D-I-S-spezifische Ausfälle d. s.

Bestimmungen ohne on-line verwertbares Ergebnis am SMA 12-60 $(n=9616)$

\begin{tabular}{|c|c|c|c|}
\hline $\begin{array}{c}\text { Kein verwertbares Ergebnis } \\
{[\%]}\end{array}$ & Gesamt & $\begin{array}{l}\text { Probe } \\
1-80\end{array}$ & $\begin{array}{l}\text { Probe } \\
81 \text {-Ende }\end{array}$ \\
\hline 1. Kreatinin & 2,7 & 3,0 & 2,4 \\
\hline 2. Harnstoff & 1,8 & 0,9 & 3,1 \\
\hline 3. Harnsäure & 4,3 & 5,4 & 2,9 \\
\hline 4. Phosphor & 6,7 & 4,6 & 9,7 \\
\hline 5. Calcium & 1,7 & 1,2 & 2,4 \\
\hline 6. Chlor & 9,2 & 9,6 & 8,7 \\
\hline 7. Gesamteiweiß & 2,4 & 1,4 & 3,8 \\
\hline 8. Albumin & 2,2 & 1,7 & 2,9 \\
\hline 9. Cholesterin & 6,5 & 4,2 & 9,7 \\
\hline 10. Bilirubin & 4,3 & 5,6 & 2,4 \\
\hline 11. Glucose im Serum & 2,2 & 2,1 & 2,5 \\
\hline $\begin{array}{l}\text { D-I-S-spezifische Ausfälle [\% } \\
\text { off-line verwertbar }\end{array}$ & 2,1 & 2,4 & 1,6 \\
\hline
\end{tabular}

Tab. 6

Gegenüberstellung der Präzision in der unterbrochenen Serie

\begin{tabular}{|c|c|c|c|c|c|c|c|c|c|c|}
\hline & & \multicolumn{4}{|c|}{ SMA $12-30$} & & \multicolumn{4}{|c|}{ SMA $12-60$} \\
\hline & & $n$ & $\overline{\mathbf{x}}$ & $\mathbf{s}$ & VK \% & & VK \% & s & $\overline{\mathbf{x}}$ & $\mathrm{n}$ \\
\hline 1. Kreatinin & {$[\mathrm{mmol} / 1]$} & 474 & 0,39 & 0,02 & 4,4 & $>$ & 2,7 & 0,01 & 0,44 & 611 \\
\hline 3. Harnsäure & {$[\mathrm{mmol} / 1]$} & 468 & 0,40 & 0,01 & 3,7 & $>$ & 3,4 & 0,01 & 0,38 & 582 \\
\hline 4. Phosphor & {$[\mathrm{mmol} / \mathrm{l}]$} & 400 & 1,75 & 0,07 & 3,7 & $>$ & 2,1 & 0,04 & 1,89 & 665 \\
\hline 5. Calcium & {$[\mathrm{mmol} / \mathrm{l}]$} & 405 & 2,38 & 0,07 & 2,9 & & 2,5 & 0,06 & 2,48 & 593 \\
\hline 6. Chlorid & {$[\mathrm{mmol} / \mathrm{l}]$} & 433 & 102,9 & 2,7 & 2,6 & $>$ & 2,0 & 2,1 & 105,7 & 657 \\
\hline 7. Gesamteiweiß & {$[\mathrm{g} / 1]$} & 471 & 66,9 & 1,6 & 2,4 & $>$ & 2,0 & 1,3 & 68,0 & 595 \\
\hline 8. Albumin & {$[\mathrm{g} / \mathrm{l}]$} & 469 & 41,4 & 1,0 & 2,4 & & 2,9 & 1,1 & 37,7 & 594 \\
\hline 9. Cholesterin & [mmol/1] & 464 & 5,19 & 0,15 & 2,9 & $<$ & 4,8 & 0,25 & 5,21 & 622 \\
\hline $\begin{array}{l}\text { 10. Bilirubin } \\
\text { 11. Glucose im }\end{array}$ & {$[\mathrm{mmol} / 1]$} & 478 & 0,033 & 0,002 & 6,8 & & 6,6 & 0,002 & 0,032 & 624 \\
\hline Serum & {$[\mathrm{mmol} / \mathrm{l}]$} & 460 & 13,3 & 0,4 & 3,4 & $p \leqq 0,05$ & 2,1 & 0,3 & 14,1 & 613 \\
\hline
\end{tabular}


Aufgeführt werden zunächst Messungen ohne verwertbare Meßpunkte. Dem stehen die D-I-S-spezifischen Ausfälle (letzte Zeile in Tab. 7) gegenüber, bedingt durch fehlende Identifizierung einer Probe wegen Kartenlesefehlern, Lesen falscher Verdünnungsfaktoren oder - als seltenste Ursache - vollkommenen Computerausfall. Bei diesen D-I-S-spezifischen Fehlern werden prinzipiell alle Kanäle gleichzeitig betroffen. Eine Auswertung der Ergebnisse erfolgt dann, als sei der SMA 12-60 nicht an einen Rechner angeschlossen. Sie werden anschließend off-line, das heißt durch manuelles Ablochen, in den Computer eingegeben.

Wie aus der Tabelle ersichtlich, treten diese D-I-S-spezifischen Fehler bei den ersten 80 Proben, die während eines Arbeitstages bestimmt werden, eher häufiger auf, als in der zweiten Tageshälfte. Dies ist bedingt durch die Kontrollen der im Nacht- und Sonntagsdienst angeforderten Untersuchungen, die gleich am Morgen danach durchgeführt werden, aber keine permanent wirksame Kurzlochkartenidentifizierung besitzen.

Geräte- oder methodenspezifisch sind die Ausfälle, die kein verwertbares Ergebnis liefern. Am anfälligsten erscheint der Chloridkanal, gefolgt vom Phosphor- und Cholesterinkanal: Am günstigsten verhalten sich Harnstoff, Calcium, Gesamteiweiß, Albumin und Serumzucker, die alle unter 2,5\% Anfälligkeit liegen.

Hinsichtlich der Unterteilung in die Gruppen Probe 1-80 und restliche Proben (das heißt 80-Ende) fällt auf, daß der Harnstoff-, Phosphor-, Gesamteiweiß- und Cholesterinkanal in der zweiten Hälfte des Tages deutlich unzuverlässiger werden, während vor allem Harnsäure und Bilirubin sich auffällig stabilisieren.

Beim Vergleich D-I-S-spezifischer Ausfälle und methoden - beziehungsweise laborgerätebedingter Ausfälle schneidet der Rechner erwartungsgemäß eher besser ab.

Eine Aufschlüsselung in eine Reihe von Ausfallursachen am SMA 12-60 wurde in Tabelle 8 versucht.

Spalte I. Ein Verstopfen des Gesamtsystems ist fastimmer durch ein Nachgerinnen der Serumprobe bedingt und betrifft naturgemäß alle Kanäle. Es läßt sich durch sorgfältiges Zentrifugieren in Grenzen halten.
Spalte II. Verstopfen eines Einzelkanals infolge differenter Reagenzbeimengung ist die Ursache für das schlechte Abschneiden der Cholesterinbestimmung. Je länger das Gerät in Betrieb ist, um so mehr tritt dieser Fehler in den Vordergrund.

Dieser Befund ist auch von anderen Seiten mitgeteilt worden (7), einschließlich.der Versuche, diesem Mangel abzuhelfen.

Auch wir haben die Probeneinleitung in das LIEberMANN-BURCHARD-Reagenz verändert, da es an dieser Stelle immer wieder zu einem Verschluß der Probenzuleitung kommt. Letztendlich sind alle Versuche unzureichend geblieben, und wir behelfen uns jetzt, indem nach jeweils 80 Seren das Gerät gestoppt und die Probeneinleitung gesäubert wird. Erst dann werden die nächsten Bestimmungen durchgeführt. Diese Störquelle harrt noch einer konstruktiv einwandfreien Beseitigung.

Spalte III. Unter technisch gerätebedingten Ursachen haben wir zusammengefaßt

1. mangelhaftes Blasenmuster,

2. defekte Dialysiermembranen,

3. defekte Pumpenschläuche,

4.- defekte im übrigen Schlauchsystem,

5. defekte in der Reagenzienzufuhr und

6. falsches Phasing.

Auffällig ist die Einlaufzeit, die Harnsäure- und Bilirubinmethode benötigen und die Instabilität der Phosphormethode. Letztere ist hauptsächlich bedingt durch Schäden an der Dialysiermembran.

Spalte IV. Eine Methode gilt dann als , außer Kontrolle;, wenn der im Testserum bestimmte Wert außerhalb der durch das Kontrollkartenprogramm festgelegten Grenzen zu liegen kommt. Deutlich über der Erwartung liegen Phosphor- und Cholesterinmethode besonders in der 2. Tageshälfte. Wahrscheinlich gerieten die Methoden aus den oben genannten gerätebedingten Gründen außer Kontrolle, ohne daß die Ursache in diesen Fällen erkannt wurde.

Die Elektrolyte werden in unserem Laboratorium einschließlich Chlorid an einem anderen Arbeitsplatz

Tab. 8

Aufschlüsselung der Fehlerursachen am SMA 12-60. Leere Positionen bedeuten 0\% Kein verwertbares Ergebnis (\%) wegen:

\begin{tabular}{|c|c|c|c|c|c|c|c|c|c|}
\hline \multirow[b]{2}{*}{ Probenposition } & \multicolumn{2}{|c|}{$\begin{array}{c}I \\
\text { Verstopfen des } \\
\text { Gesamtsystems }\end{array}$} & \multicolumn{2}{|c|}{$\begin{array}{c}\text { II } \\
\text { Verstopfen eines } \\
\text { Einzelkanals }\end{array}$} & \multicolumn{2}{|c|}{$\begin{array}{c}\text { III } \\
\text { Ursache techn. } \\
\text { gerätebēdingt }\end{array}$} & \multicolumn{2}{|c|}{$\begin{array}{c}\text { IV } \\
\text { Methode außer } \\
\text { Kontrolle }\end{array}$} & \multirow[t]{2}{*}{$\begin{array}{c}\text { V } \\
\text { Ursache } \\
\text { unklar }\end{array}$} \\
\hline & $1-80$ & $>80$ & $1-80$ & $>80$ & $1-80$ & $>80$ & $1-80$ & $>80$ & \\
\hline 1. Kreatinin & 0,8 & 1,4 & & & 1,3 & 0,2 & 0,4 & 0,5 & 0,5 \\
\hline 2. Harnstoff & 0,8 & 1,4 & & & & 0,2 & & 0,5 & 0,5 \\
\hline 3. Harnsäure & 0,8 & 1,4 & & & 2,3 & 0,2 & 1,1 & 1,0 & 0,8 \\
\hline 4. Phosphor & 0,8 & 1,4 & 0,8 & & 1,2 & 3,6 & 1,3 & 2,0 & 1,4 \\
\hline 5. Calcium & 0,8 & 1,4 & & & 0,4 & 0,2 & & 0,5 & 0,1 \\
\hline 6. Chlor & 0,8 & 1,4 & & & 0,1 & 0,3 & 5,7 & 3,1 & 2,3 \\
\hline 7. Gesamteiweiß & 0,8 & 1,4 & & & 0,2 & 0,4 & 0,3 & 1,0 & 0,5 \\
\hline 8. Albumin & 0,8 & 1,4 & & & 0,2 & 0,2 & 0,4 & 1,0 & 0,3 \\
\hline 9. Cholesterin & 0,8 & 1,4 & 1,4 & 2,6 & 0,5 & 0,4 & 2,4 & 2,4 & 1,7 \\
\hline 10. Bilirubin & 0,8 & 1,4 & 0,5 & & 2,8 & 0,2 & 1,3 & 0,5 & 0,3 \\
\hline 11. Glucose im Serum & 0,8 & 1,4 & & & 0,2 & 0,3 & & 0,5 & 0,7 \\
\hline
\end{tabular}


bestimmt. Chlorid läuft im SMA $12-60$ in den meisten Fällen ohne Anforderung mit. Deshalb wird der Chloridwert vermutlich zu häufig ohne Grund gestrichen und auf den am anderen Arbeitsplatz erstellten Wert zurückgegriffen. Dieses Vorgehen erklärt das schlechte Abschneiden der Chloridmessung im SMA 12-60.

Dieser Verdacht erhärtet sich auch bei der Betrachtung der Spalte V der Tabelle 8, die den Prozentsatz einzelner, nicht plausibler Herausschläger aus unklarer Ursache umfaßt. Neben der Chlorid- liegen hier die Cholesterin- und Phosphormethode an der Spitze der Anfälligkeit.

Alle diese Bestimmungen ohne verwertbares Ergebnis, aus welcher Ursache immer, in jedem Fall aber geräteoder methodenbedingt, müssen ein zweites Mal durchgeführt werden und schränken die Effektivität des SMA $12-60$ unter die 71,4 bis $75,5 \%$ der Tabelle 2 ein. Es resultieren dann für den SMA 12-60 zwischen 21,4 bis 29,0 analysierte Patientenseren pro $h$ unter Berücksichtigung von ẹtwa $7 \%$ vergebens durchgeführter Bestimmungen.

\section{Bewertung der Ergebnisse und Schlußfolgerungen}

Die wesentlichen Unterschiede des SMA 12-60 Analysengerätes der Firma Technicon gegenüber dem Vorgängertyp SMA 12-30 im Diagnostik-InformationsSystem unserer Klinik liegen

1. in der Kapazitätserhöhung, die sich in der Steigerung von 126 auf 210 theoretisch mögliche Proben pro $8 \mathrm{~h}$ mit 11 bzw. 12 Bestimmungen ausdrückt,

2. in der auf $1,8 \mathrm{ml}$ verminderten Serummenge gegenüber 2,8 $\mathrm{ml}$ beim SMA 12-30,

3. in der signifikanten Verbesserung der Präzision in der unterbrochenen Serie bei 6 von 11 Methoden, klinisch relevant zumindest bei der Kreatininmethode. 9 der 11 Methoden haben einen Variationskoeffizienten von deutlich unter $3,8 \%$ (nämlich zwischen $1,8 \%$ und $3,4 \%$ ). Dies bedeutet, daß in 99 von 100 wiederholten Bestimmungen die Abweichung zum erstbestimmten Wert weniger als $10 \%$ beträgt.

\section{Einen Rückschritt bedeutet}

4. die Anfälligkeit der Cholesterinmethode im SMA 12-60. In der ersten Tageshälfte müssen $4,2 \%$, in der zweiten Tageshälfte $9,7 \%$ aller Bestimmungen wiederholt werden, wenn man auf den Cholesterinwert nicht verzichten will. Zugleich verschlechtert sich der Variationskoeffizient (in der unterbrochenen Serie) von $2,9 \%$ beim SMA $12-30$ auf $4,8 \%$ beim neuen Gerät.
Aus dem on-line Anschluß der Geräte an den Rechner IBM 1800 erwachsen

5. im Vergleich zu den gerätebedingten nur untergeordnete Fehler. Sie beschränken sich auf mangelnde Identifizierung der Analysenergebnisse. Dies ist bedingt durch Kurzkartenlesefehler oder durch fehlende Kurzkarten bei Notfällen. Durch manuelles Ablochen werden diese „Ausfälle“ in den Rechner eingegeben. Insgesamt betreffen diese Fehler nur 2,1\% der gesamten Resultate und stellen damit keine Belastung des Organisationsablaufs dar. Man muß aber bedenken, daß diese konventionelle off-line Eingabe fehleranfällig ist. Sowohl beim Ablesen der Werte vom Schreiber des Gerätes, wie beim Schreiben, beim Lesen dieser Listen und beim Ablochen sind Übertragungsfehler nicht ausgeschlossen und praktisch, bis auf Einzelfälle, nicht zu entdecken.

Bei der on-line Verarbeitung der Daten sind diese Fehler ausgeschlossen. Es ist noch nie zur Übertragung falscher Analogwerte am SMA 12 gekommen. Die Digitalwertübertragung ist entweder exakt oder ein Kartenfehler (Lesefehler) wird erkannt, eine andere Möglichkeit ist prinzipiell ausgeschlossen. Während der 70 tägigen Überwachungsperiode zur Fehleranalyse kam es nie zu einem vollständigen Rechnerausfall.

6. Der SMA 12-60 im Rahmen des D-I-S Tübingen liefert etwa $25 \%$ der etwa 6000 numerischen Laborergebnisse pro Tag.

7. Eine Dokumentation der Ergebnisse einschließlich der Präzisionsüberwachung macht im D-I-S keine Mehrarbeit. Bei voll- oder teilmechanisierten Meßverfahren muß man mit einem Aufwand von über 50\% Schreibarbeit an der Gesamtarbeitszeit der technischen Assistenzkräfte rechnen. Diese Arbeit wird im D-I-S Tübingen vollständig vom Rechner übernommen.

8. Die mit eineinhalb Arbeitskräften pro Arbeitstag mit dem SMA 12-60 geleistete Probenfrequenz beträgt zur Zeit im Durchschnitt 146 Proben bzw. 1573 Analysenresultate gegenüber vielleicht 50 bis 150 Analysenresultaten pro Person bei konventionellen Verfahren. (Die maximale Tagesleistung liegt zur Zeit bei 270 Proben ohne Test- und Kontrollpositionen.)

9. Legt man auf ein vollständiges Analysenmuster in der bearbeiteten Probe Wert, so sind (laut der Anfälligkeit in Tab. 7) etwa 7\% aller Bestimmungen zu wiederholen. Auch dann fehlt im Durchschnitt in jedem 10. untersuchten Patientenserum eines der 11 Ergebnisse.

\section{Danksagung}

Der Stiftung Volkswagenwerk sind wir zu Dank verpflichtet. Mit ihren Mitteln wurde die Installation der Computeranlage ermöglicht. 


\section{Literatur}

1. Schily, K., Eggstein, M., KNodel, W., Allner, R., Henig, S. \& Kuhlmann, E. (1969), diese Z. 7, 66-71. - 2. Graeser, W. \& Schmülling, R.-M. (1970), Erfahrungen mit on-line angeschlossenem Standard-Auto-Analyzer und SMA 12-30 im Diagnostik-Informations-System Tübingen. GrAESER, W. On-line Meßwerterfassung, Probenidentifizierung und Auswertung. SCHMÜlling, R.-M. Analysenspektrum und Labororganisation. Technicon Symposium 70 Bad Homburg V.D.H. (1970).-3.Bock, H. E. \& EgGSTEIN, M. (1970), Diagnostik-Informations-System. Integrierte elektronische Datenverarbeitung für die ärztliche Diagnostik. Springer-Verlag, Berlin-Heidelberg-New York. -
4. Bürtiner, H., Hansert, E. \& Stam, D. (1970), In Methoden der enzymatischen Analyse. Band I. S. 329-364. Bergmerer, H. U., Herausgeber. Verlag Chemie GmbH, Weinheim, Bergstr. 5. HaLd, A. (1952), Statistical theory with engeneering applications. J. Wiley and Sons Inc., New York. - 6. BeNNETr, C. A. \& FrankLIN, N. L. (1967), Statistical analysis in chemistry and the chemical industry. John. Wiley and Sons, Inc. New York-LondonSydney fifth printing. - 7. Gossler, O.; Der SMA 12-60 im Zentrallaboratorium eines Krankenhauses. Technicon-Symposium 71. - 8. Eggstein, M., Allner, R., KuhlmanN, E. \& KNodel, W. (1966), Klin. Wochenschr. 44, 424-430.

Dr. med. R.-M. Schmülling Prof. Dr. med. M. Eggstein 74 Tübingen

Medizinische Universitätsklinik Otfried-Müller-Straße 